\title{
Imaging of myocardial diseases: to the new horizons
}

\author{
Valentin Sinitsyn* \\ Federal Center of Treatment and Rehabilitation, Ivan'kovsloye sh.3, Moscow, 125367, Russia
}

This special issue of Clinical Case Reports and Reviews is devoted to rare cases of different myocardial diseases. It is well-known that oninvasive cardiac imaging is a cornerstone of the diagnosis of most cardiac diseases and it is extremely helpful in rare cardiovascular pathologies. Different forms of cardiomyopathies, acute and chronic myocarditis, the secondary involvement of the heart in different congenital and systemic diseases are very difficult to diagnose.

Cardiac imaging - first of all, CT, MRI and hybrid modalities offers unique opportunities for detection and characterization of these pathologies. Some decades ago many of modern cardiac diseases were unknown to medical specialists. A good example is cardiomyopathies. They have been described just in 70-ies of XX century and progress in their detection and characterization was substantially related to the revolutionary technical developments of modern diagnostic cardiac imaging modalities Today cardiac radiology provides new insights both into myocardial structure,function and risk stratification and patient's prognosis. This issue shows how the traditional and new cardiac imaging tools - such as late-enhancement MRI, T1- and T2mapping, low-dose CT-angiography and dual-energy CT help to detect and characterize some rare cardiac diseases.

Developments in cardiac imaging are quick and spectacular. Diagnostic opportunities of all imaging modalities are changing rapidly so some questions about proper choice of imaging modality sometimes arise and give ground to further research and comparative studies. Is T1-mapping or late-enhancement MRI equally sensitive to detect focal or diffuse myocardial inflammation of fibrosis? Could delayed myocardial CT be used as a substitute to MR delayed enhancement with gadolinium? How can cardiac imaging help to differentiate between primary and secondary cardiomyopathies?

Articles published in this special issue give some insights into these and some other diagnostic challenges in cardiovascular imaging. So far indications for use of new diagnostic modalities (i.e., T1-mapping, CT myocardial delayed enhancement) are not yet included in current guidelines but there is more and more evidence that they could be very useful in some clinical situations. We hope that these cases would be interesting for readers and stimulate further interest to research in this intriguing field.
Copyright: (C)2016 Sinitsyn V. This is an open-access article distributed under the terms of the Creative Commons Attribution License, which permits unrestricted use, distribution, and reproduction in any medium, provided the original author and source are credited.
Correspondence to: Dr. Valentin Sinitsyn, Federal Center of Treatment and Rehabilitation, Ivan'kovsloye sh.3, Moscow, 125367, Russia, Tel: +7 495 9424020; E-mail: vsini@mail.ru

Special Issue: Imaging of myocardial diseases: to new horizons

Valentin E. Sinitsyn

Professor

Department of Radiology

Federal Center of Medicine and Rehabilitation

Russia

E-mail: vsini@mail.ru

Published: October 18, 2016 